\title{
Role of Glucose Breath Test for Small Intestinal Bacterial Overgrowth in Children and Adolescents With Functional Abdominal Pain Disorders in Korea
}

\author{
Jin Lee, ${ }^{1}$ Chang-Nyol Paik, ${ }^{2 *}$ Dae Bum Kim, ${ }^{2}$ Ji Min Lee, ${ }^{2}$ Yeon-Ji Kim, ${ }^{2}$ Sik Kyung Choi, ${ }^{3}$ and Yeon Jong Cho ${ }^{3}$ \\ ${ }^{I}$ Department of Pediatrics, Incheon St. Mary's Hospital, College of Medicine, The Catholic University of Korea, Seoul, Korea; ${ }^{2}$ Department of \\ Internal Medicine, St. Vincent's Hospital, College of Medicine, The Catholic University of Korea, Seoul, Korea; and ${ }^{3}$ Department of Pediatrics, \\ Hanil General Hospital, Seoul, Korea
}

\section{Background/Aims}

Small intestinal bacterial overgrowth (SIBO) is expected in children and adolescents with functional abdominal pain disorders (FAPDs). This study is conducted to estimate the prevalence of SIBO and to investigate the role of SIBO in children and adolescents with FAPDs.

\section{Methods}

This prospective study enrolled children with FAPDs fulfilling the Rome IV criteria. A hydrogen-methane glucose breath test was used to diagnose SIBO. A survey of bowel symptoms using questionnaires, birth history, types of feeding, and the presence of allergy was conducted.

\section{Results}

Sixty-eight children and adolescents (range, 6-17 years; median, 12.5 years) were enrolled. SIBO was detected in 14 patients (20.6\%). Age ( $\geq 12$ years) $(P<0.003)$ and loose stool $(P=0.048)$ were significantly more common in children with SIBO than in children without SIBO. However, the history of allergies $(P=0.031)$ was less common in children with SIBO than those without SIBO. No significant differences were observed in other demographic findings. In multivariate analysis, age ( $\geq 12$ years) was the independent factor predicting SIBO in children with FAPDs.

\section{Conclusions}

SIBO is not uncommon in children and adolescents with FAPDs. Among children aged above 12 years and diagnosed with FAPDs, SIBO is a suspected clinical target for treatment to relieve intestinal symptoms. A further study to investigate the association between intestinal bacteria and history of allergy is needed.

(J Neurogastroenterol Motil 2022;28:78-85)

Key Words

Adolescent; Child; Functional abdominal pain disorders; Glucose breath test; Small intestinal bacterial overgrowth

Received: October 11, 2020 Revised: January 15, 2021 Accepted: January 28, 2021

(.) This is an Open Access article distributed under the terms of the Creative Commons Attribution Non-Commercial License (http://creativecommons. org/licenses/by-nc/4.0) which permits unrestricted non-commercial use, distribution, and reproduction in any medium, provided the original work is properly cited.

*Correspondence: Chang-Nyol Paik, MD, PhD Division of Gastroenterology, Department of Internal Medicine, St. Vincent's Hospital, College of Medicine, The Catholic University of Korea, 93 Jungbu Daero, Paldal-gu, Suwon, Gyeonggi-do 16247, Korea Tel: +82-31-881-8582, Fax: +82-31-253-8898, E-mail: cmcu@catholic.ac.kr 


\section{Introduction}

The symptoms of functional abdominal pain disorders (FAPDs) based on Rome IV criteria, previously referred to as abdominal pain-related functional gastrointestinal disorders, represent some of the most common gastrointestinal complaints, and affect up to $20 \%$ of children in Western countries. ${ }^{1,2}$ Although the pathophysiological mechanisms of FAPDs are still poorly understood and any definitive treatment has yet to be established, altered gastrointestinal motility, hypersensitivity to bowel distension, impaired brain-gut interaction, intestinal dysbiosis, psychosocial problem, and immune mechanism have been suggested as possible multifactorial mechanisms. Recently, small intestinal bacterial overgrowth (SIBO) has been cited as one of the etiologic factors for FAPDs, demonstrated by a breath test. ${ }^{3,4}$

SIBO is defined as an increase in the number of bacteria in the small intestine. Jejunal aspirate culture is considered a gold standard test for SIBO diagnosis. However, this diagnostic method is invasive, expensive, and uncomfortable. Thus recently, a noninvasive breath test has been preferred, because of its simplicity and lower expense. The increase in hydrogen $\left(\mathrm{H}_{2}\right)$ excretion is generally considered a diagnosis of SIBO in clinical practice. However, nearly $30 \%$ of the general population carries mostly intestinal methanogens and produces methane $\left(\mathrm{CH}_{4}\right)$ as a major by-product of carbohydrate fermentation. ${ }^{5}$ Therefore, adding $\mathrm{CH}_{4}$ to $\mathrm{H}_{2}$ measurement may avoid false-negative results. Although the relatively high prevalence of SIBO in children with functional gastrointestinal disorder (FGID) was suggested, there is a paucity of evidence involving children with FAPDs based on concurrent $\mathrm{H}_{2}$ and $\mathrm{CH}_{4}$ breath tests.

The study estimated the prevalence of SIBO using a glucose $\mathrm{H}_{2}-\mathrm{CH}_{4}$ breath test and investigated the clinical role of SIBO in children with FAPDs.

\section{Materials and Methods}

The protocol was approved by the Institutional Review Boards of Hanil General Hospital of Korea (IRB No. HIRB-2017-003). The study was conducted in compliance with the tenets of the Declaration of Helsinki and International Committee on Harmonization Guidelines for Good Clinical Practice. A written Informed consent was obtained from all children or the authorized legal representatives prior to enrolling the subjects in the study.

\section{Study Population}

The study was conducted at a secondary teaching hospital in Seoul, Korea, from July 2017 to April 2020. We enrolled consecutive children who visited the institution, fulfilling the Rome IV criteria for FAPDs between 6 years to 18 years of age. The subjects voluntarily participated in the study and their guardians agreed to participate. Exclusion criteria were a history of diabetes, thyroid disease, connective tissue disease, inflammatory bowel disease, or gastrointestinal or gynecologic surgery. Children who were treated with antisecretory agents such as proton pump inhibitors or histamine $\mathrm{H} 2$ receptor blockers, antibiotics, probiotics, prokinetics, narcotics, laxatives, bulking agents, or antidiarrheal agents within the previous 4 weeks; and those with renal insufficiency, major psychiatric problem, hearing disturbance, masticatory dysfunction, and incomplete data, or those who had underwent colonoscopy within the last 3 months, were also excluded.

\section{Study Design}

The survey design encompassed the clinical demographics, type of feeding (breast milk or formula) during infancy, type of delivery (vaginal delivery or Cesarean section), and history of allergy of all the enrolled children. Bowel symptoms were surveyed using a questionnaire, and glucose $\mathrm{H}_{2}-\mathrm{CH}_{4}$ breath test was used for the diagnosis of SIBO. The children were presented to the pediatric clinic after a minimum of 12 hours of fasting before the test. Physical exercises was not allowed during the test, starting 30 minutes before the test. The end expiratory breath samples were collected at baseline after ingestion of oral glucose solution in $2 \mathrm{~g} / \mathrm{kg}$ (maximum 50 g) (DIASOL-S SOLN; Tae Joon Pharma, Seoul, Korea), and then for 120 minutes at every 10-minute intervals. Breath tests of the samples with breath test kit (The EasySampler Breath Test Kit; Quintron Instrument Company, Milwaukee, WI, USA) were performed each time by gas chromatography equipment (Quintron BreathTracker SC; Quintron Instrument Company).

\section{Definition of Small Intestinal Bacterial Overgrowth and Assessment of Intestinal Symptom}

A positive glucose breath test (GBT) indicating SIBO was defined and classified as follows ${ }^{6,7}$ : (1) an increase in $\mathrm{H}_{2}$ concentration of more than 12 ppm above baseline within 90 minutes (glucose $\mathrm{H}_{2}$ breath test [GHBT+] group) or (2) an increase in $\mathrm{CH}_{4}$ concentration of more than $10 \mathrm{ppm}$ above baseline within 90 minutes (glucose $\mathrm{CH}_{4}$ breath test [GMBT+] group). The GBT (mixed)+ group was defined when both conditions (GHBT and GMBT 
groups) were met. All participants completed the bowel symptom questionnaire. The Korean version of the Irritable Bowel Syndrome Quality of Life questionnaire was based on the Rome criteria together with additional questions about gastro intestinal symptoms, and has been verified and used in previous studies., ${ }^{8,9}$ Besides, 13 questions related to various gastrointestinal symptoms experienced in the last 4 weeks were included. Information on bowel symptoms of discomfort, pain, hard or lumpy stools, loose or watery stools, straining during bowel movements, bowel urgency, mucus passing during bowel movements, abdominal fullness/bloating or swelling, flatus, chest pain or heartburn, the feeling of being full soon after a meal, urinary frequency, and nausea was collected. The severity of symptoms was estimated by the total symptom score, defined by the cumulative scores of event frequency and intrusiveness. The frequency and intrusiveness of each symptom were evaluated by each patient using a 7-point scale from 0 to 6 . Since the total symptom score was defined as the sum of the symptom frequency and intrusiveness scores, the range of a score by each symptom was 0-12.

\section{Statistical Methods}

Clinical evaluations included age, sex, body mass index (BMI), types of feeding or delivery, allergy history, intestinal symptoms, and presence of irritable bowel syndrome (IBS) according to the pres-

Table 1. Baseline Characteristics of Children and Adolescents With Functional Gastrointestinal Disorders $(\mathrm{N}=68)$

\begin{tabular}{lc}
\hline \multicolumn{1}{c}{ Demographics } & \\
\hline Age $(\mathrm{yr})$ & $12.24 \pm 2.94$ (range 6-17) \\
Gender & $25(36.8)$ \\
Male & $43(63.2)$ \\
Female & $152.35 \pm 14.60$ \\
Height $(\mathrm{cm})$ & $46.00 \pm 15.18$ \\
Weight $(\mathrm{kg})$ & $19.27 \pm 3.56$ \\
BMI $\left(\mathrm{kg} / \mathrm{m}^{2}\right)$ & $26(38.2)$ \\
Types of feeding & $46(25.0)$ \\
Breast milk & $25(36.8)$ \\
Formula & \\
Mixed & $42(61.8)$ \\
Types of delivery & $26(38.2)$ \\
Vaginal delivery & $20(29.4)$ \\
Cesarean section & $14(20.6)$ \\
Allergy history & $6(8.8)$ \\
Positive GBT & $8(11.8)$ \\
$\mathrm{H}_{2}$ &
\end{tabular}

BMI, body mass index; GBT, glucose breath test; $\mathrm{H}_{2}$, hydrogen; $\mathrm{CH}_{4}$, methane.

Data are expressed as mean $\pm \mathrm{SD}$ or $\mathrm{n}(\%)$. ence of SIBO. The profiles of breath $\mathrm{H}_{2}$ and $\mathrm{CH}_{4}$ in children with FAPDs were compared with those of historically healthy controls who were previously registered in the determination of normal GBT value at the Catholic University of Medicine. ${ }^{10}$ Continuous data were expressed as mean $\pm \mathrm{SD}$ and were analyzed using independent-sample $t$ tests, whereas the categorical variables were expressed as quantities and analyzed using $\chi^{2}$ tests. Multiple stepwise logistic regression was used to identify independent factors correlated with the existence of the SIBO. A $P$-value less than 0.05 was considered significant for all tests.

\section{Results}

\section{Study Populations}

Seventy children enrolled in the study. Two subjects were excluded from the analysis due to a sampling error, and incompletion of demographic data or bowel symptom questionnaire. Baseline characteristics including demographics and type of feeding, delivery and allergy are shown in Table 1. Twenty children were allergic, and involved several types of allergy: atopic dermatitis $(\mathrm{n}=5)$, allergic rhinitis $(n=4)$, pollen allergy $(n=2)$, food allergy involving mushroom ( $\mathrm{n}=1)$ or fruits such as apple, kiwi, and watermelon ( $\mathrm{n}$ $=2)$, insect allergy $(\mathrm{n}=1)$, dust allergy $(\mathrm{n}=1)$, cold allergy $(\mathrm{n}=$ $1)$, urticaria $(n=1)$, asthma with allergic rhinitis $(n=1)$, allergic rhinitis with atopic dermatitis ( $\mathrm{n}=1)$, or cat allergy combined with allergy to nuts $(\mathrm{n}=1)$.

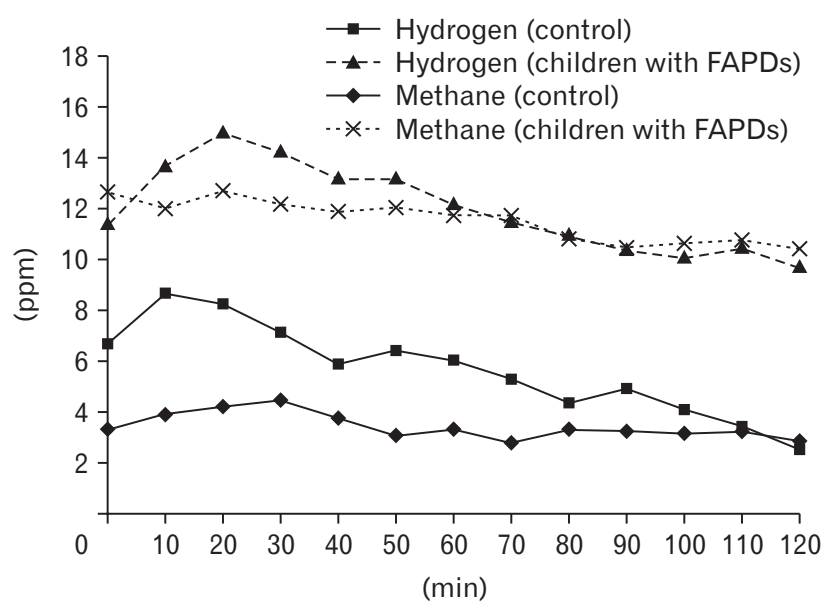

Figure 1. Comparison of breath hydrogen and methane profiles (parts per million [ppm]) between historic controls and children with functional abdominal pain disorders (FAPDs). 


\section{Glucose Breath Test in Children With Functional Abdominal Pain Disorders and Healthy Controls}

Fourteen of 68 children (20.6\%) were positive in the GBT; $6(8.8 \%)$ excreted $\mathrm{H}_{2}$, and $8(11.8 \%)$ were combined excreters, whereas none was included in the $\mathrm{CH}_{4}$ excreters (Table 1). There were significant differences in the exhaled $\mathrm{H}_{2}$ at intervals of 0,30 , 50, 60, 70, 80, 100, 110, and 120 minutes, and in $\mathrm{CH}_{4}$ from 10 to 120 minutes intervals between the children with FAPDs and controls, respectively (Fig. 1).

\section{Characteristics of Children With Functional Abdominal Pain Disorders According to Glucose Breath Test Positivity}

No difference was found between GBT positive $(\mathrm{GBT}+)$ and negative (GBT - ) children regarding gender, BMI, types of feeding, and types of delivery. In the GBT + children with FAPDs, the age was significantly higher whereas the presence of allergy histories was significantly lower than those of GBT - children with FAPDs

Table 2. The Characteristics of Children With Functional Gastrointestinal Disorders to the Positivity to Glucose Breath Test

\begin{tabular}{|c|c|c|c|}
\hline \multirow{2}{*}{ Demographics } & \multicolumn{2}{|c|}{ GBT } & \multirow{2}{*}{$P$-value } \\
\hline & Negative $(n=54)$ & Positive $(\mathrm{n}=14)$ & \\
\hline Age (yr) & $11.91 \pm 3.00$ & $13.50 \pm 2.38$ & 0.070 \\
\hline$<12$ & $32(59.3)$ & $2(5.9)$ & 0.003 \\
\hline$\geq 12$ & $22(40.7)$ & $12(85.7)$ & \\
\hline \multicolumn{4}{|l|}{ Gender } \\
\hline Male & $20(37.0)$ & $5(35.7)$ & 0.927 \\
\hline Female & $34(63.0)$ & $9(64.3)$ & \\
\hline $\operatorname{BMI}\left(\mathrm{kg} / \mathrm{m}^{2}\right)$ & $18.88 \pm 3.27$ & $20.75 \pm 4.34$ & 0.080 \\
\hline \multicolumn{4}{|l|}{ Types of feeding } \\
\hline Breast milk & $24(44.4)$ & $2(14.3)$ & 0.079 \\
\hline Formula & $11(20.4)$ & $6(42.9)$ & \\
\hline Mixed & $19(35.2)$ & $6(42.9)$ & \\
\hline \multicolumn{4}{|l|}{ Types of delivery } \\
\hline Vaginal delivery & $32(59.3)$ & $10(71.4)$ & 0.404 \\
\hline Cesarean section & $22(40.7)$ & $4(28.6)$ & \\
\hline \multicolumn{4}{|l|}{ Allergy history } \\
\hline No & $35(64.8)$ & $13(92.9)$ & 0.031 \\
\hline Yes & $19(35.2)$ & $1(7.1)$ & \\
\hline \multicolumn{4}{|l|}{ IBS } \\
\hline No & $38(70.4)$ & $6(42.9)$ & 0.056 \\
\hline Yes & $16(29.6)$ & $8(57.1)$ & \\
\hline
\end{tabular}

GBT, glucose breath test; BMI, body mass index; IBS, irritable bowel syndrome.

Data are expressed as mean $\pm \mathrm{SD}$ or $\mathrm{n}(\%)$.
(Table 2). In multivariable logistic regression, age $\geq 12$ was the only significant independent factor predicting SIBO in children with FAPDs. A history of allergy inversely correlated with SIBO with statistically significant tendency (Table 3 ). The breath $\mathrm{H}_{2}$ profiles at all the time points were significantly higher in children with a history of allergy compared with those without (Fig. 2).

\section{Bowel Symptoms According to Glucose Breath Test Positivity in Children With Functional Abdominal Pain Disorders}

Although GBT + children with FAPDs had high individual total symptom scores regarding abdominal pain, hard stool, strain, urgency, bloating, flatus, satiety, frequent urination, and nausea (Fig. $3)$, the total frequency scores $(32.50 \pm 12.23$ vs $28.76 \pm 12.43$, $P=0.321)$, total bothersome scores $(24.86 \pm 15.64$ vs $21.43 \pm$ $13.34, P=0.411)$, and total sum scores (57.36 \pm 27.16 vs 50.19 $\pm 24.77, P=0.347$ ) compared to those of GBT - children with

Table 3. Multivariate Analysis for the Independent Factors of Positive Glucose Breath Test

\begin{tabular}{lrlc}
\hline \multicolumn{1}{c}{ Demographics } & OR & \multicolumn{1}{c}{$95 \%$ CI } & $P$-value \\
\hline Age $\geq 12 \mathrm{yr}$ & 11.364 & $1.621-76.923$ & 0.014 \\
BMI $\left(\mathrm{kg} / \mathrm{m}^{2}\right)$ & 1.023 & $0.819-1.276$ & 0.844 \\
The presence of allergy & 0.129 & $0.011-1.546$ & 0.106 \\
IBS & 3.390 & $0.691-16.667$ & 0.133 \\
Types of feeding & & & \\
Breast milk & 0.526 & $0.055-5.053$ & 0.578 \\
Powdered milk & 3.540 & $0.572-21.914$ & 0.174 \\
\hline
\end{tabular}

GBT, glucose breath test; BMI, body mass index; IBS, irritable bowel syndrome.

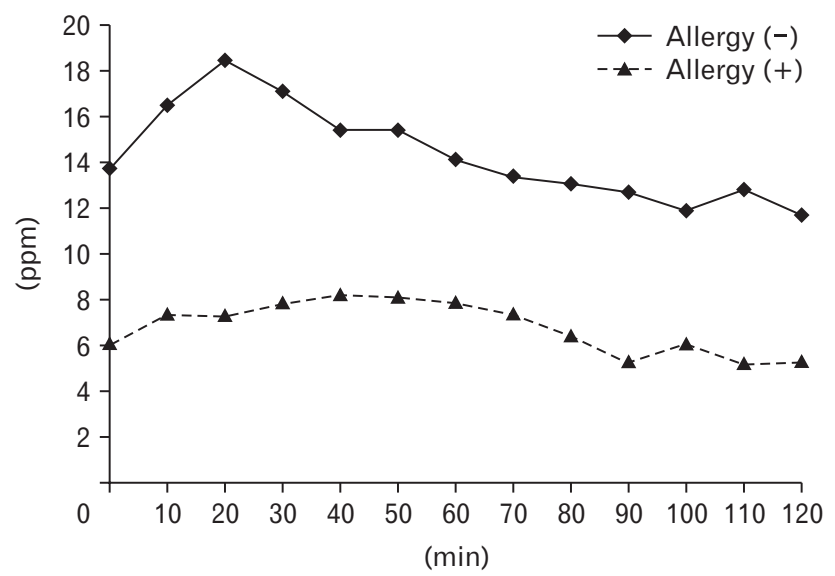

Figure 2. Profiles (parts per million [ppm]) of breath hydrogen in children with functional abdominal pain disorders (FAPDs) based on history of allergic disease. 


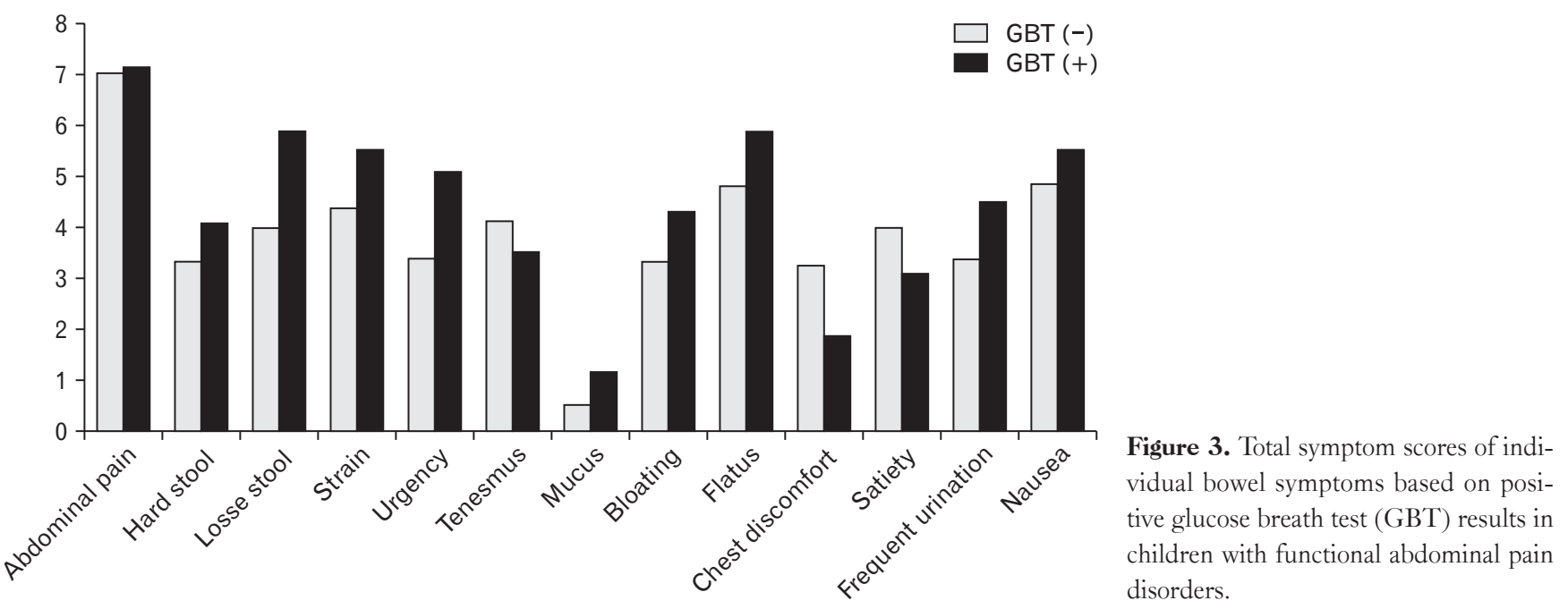

FAPDs, showed no significant differences between the 2 groups. In the GBT + group, the total symptom scores of loose stool were significantly higher than those of the GBT - group (5.86 \pm 3.03 vs $3.98 \pm 3.13, P=0.048$ ) (Fig. 3).

\section{Discussion}

The current study showed that SIBO is not uncommon in children and adolescents with FAPDs. It appears to be closely related to worsening intestinal symptoms in children older than 12 years diagnosed with FAPDs, and represents a meaningful therapeutic target for amelioration of functional intestinal symptoms.

The causes of FGID include not only SIBO but also a number of factors including altered bowel motility, visceral hypersensitivity, abnormal brain-gut interaction, autonomic dysfunction, and activation of the immune system. ${ }^{11}$ SIBO could induce functional gastrointestinal symptoms in adults or children, such as chronic abdominal pain, bloating, diarrhea, flatulence, and/or constipation., ${ }^{3,12-14}$

Direct jejunal aspirate culture is the gold standard method for diagnosing SIBO. However, this method has limitations such as difficulty in accessing the distal small bowel, possibility of contamination during sampling, low reproducibility, and potential for false negative results. ${ }^{15-17}$ It is also somewhat uncomfortable and invasive. Thus a breath test is a simple alternative and an acceptable diagnostic method for SIBO. Various factors including substrate types, criteria for test results, gas types measured $\left(\mathrm{H}_{2}\right.$ or $\left.\mathrm{CH}_{4}\right)$, and research design could directly affect the results of breath test. Accordingly, a wide variety of prevalence of SIBO has been reported. ${ }^{18,19}$ There are still controversy to usefulness for breath tests to reflect SIBO in patients with FGID ${ }^{20,21}$ In addition, most proposed guidelines ${ }^{22,23}$ for breath tests are based on research for adults, and thus it is unclear whether this proposal will also apply to children.

However, this non-invasive method may be more appropriate in children who are burdened with invasive tests. The first $\mathrm{Rome}_{\mathrm{H}_{2}}$ Breath Testing Consensus Conference Working Group (2009) recommended the GBT instead of lactulose breath test for diagnostic accuracy. ${ }^{22}$ The advantage of breath test using glucose as a substrate over lactulose is low false positivity and superior diagnostic ability of proximal SIBO ${ }^{24,25}$ In children who are expected to have a low BMI compared to that of adults, the intestinal transit time is also relatively short, and therefore, glucose was selected for breath tests. During lactulose breath tests, it is difficult to distinguish SIBO from colonic bacteria if intestinal transit is fast, ${ }^{26,27}$ and importantly lactulose itself induces rapid small intestine transit. ${ }^{28}$ The study demonstrated that the prevalence of SIBO in children with FAPDs was 20.6\% (14/68) using the $\mathrm{H}_{2}-\mathrm{CH}_{4}$ GBT, which was consistent with other study results with a prevalence of $22.5 \%$ in children. ${ }^{6}$ Only a single study ${ }^{3}$ involving children with abdominal pain-related FGIDs according to the Rome III criteria demonstrated an SIBO prevalence of $14.3 \%$ in English literature, which measured only $\mathrm{H}_{2}$ produced by SIBO, and included baseline breath $\mathrm{H}_{2} \geq 20 \mathrm{ppm}$ testing positive to GHBT. Our study evaluates both breath $\mathrm{H}_{2}$ and $\mathrm{CH}_{4}$ to detect hydrogenic and methanogenic bacteria, respectively. It is known that about $15-27 \%$ of $\mathrm{SIBO}$ patients are $\mathrm{CH}_{4}$ producers. ${ }^{5}$ The prevalence of SIBO can be underestimated without measuring breath $\mathrm{CH}_{4}$. Recently, the North American Consensus did not recommend the baseline breath profile as positivity to breath test due to the need for clarifying whether it was a result of improper test preparation or represented a SIBO variant. ${ }^{23}$ We excluded the fasting breath profiles as diagnostic criteria for SIBO, and only in- 
cluded positivity to GBT using a cutoff of increased breath profiles compared with participant baselines. ${ }^{23}$ However, these recommendations are based on studies of adults and extrapolation to children needs further investigation.

The other strength of this study is that it was conducted in Asia. The clinical use of breath test in Asia has occurred only in the last few years, being lagging in time compared to the West. The prevalence of SIBO is thought to be different between patients with Asian and Western countries due to main dietary habit, physical status, or bowel transit time. ${ }^{29-31}$ This study provided evidence that SIBO using breath test play an important role in children with functional intestinal symptoms in Asia as well as in Western children. Future studies are needed to conduct additional comparative studies on the different targeting age groups, effects of dietary habits and lifestyles between those in Asia and Western countries, or research on immigrants in Korea.

The potential limitation relates to the use of fewer historical adult controls compared with the cases. However, the breath $\mathrm{H}_{2}$ profiles at most time points during the tests involving cases were increased compared with those of adult controls as shown in Figure 1. Because age itself is a known risk factor for SIBO, the profiles of breath tests in adult controls were expected to be higher than in controls with children. ${ }^{32}$ Nevertheless, the range of prevalence of SIBO in children is still very wide due to various diagnostic cutoff values and methodologies. ${ }^{4}$ Therefore, further research is needed to verify the diagnosis of bacterial overgrowth in children by GBT.

The age of 12 years and older equivalent to that of subjects in middle school and high school is a significant predicting factor for SIBO in children and adolescents with FAPDs. SIBO seems to be more prevalent among older individuals despite studies involving adults, ${ }^{32}$ who are more likely to have reduced intestinal motility, small bowel diverticulosis, low gastric secretory function, or a strong history of medication usage. Although non-significant statistical differences in multivariate analysis were shown, a low level of BMI, the presence of IBS, or loose stool were associated with SIBO in this study. Still in debate, the prevalence of SIBO is reported to be high in patients with IBS, or chronic diarrhea. ${ }^{21}$ The prevalence of breast feeding and allergic history tended to be low in the presence of SIBO. Representative factors predicting the status of gut microbiota during infancy include the types of delivery and feeding practices. A study suggested that dysbiosis was significantly related to lack of breastfeeding, but showed no evidence of association with mode of delivery, ${ }^{33}$ which is consistent with our results. However, the relationship between breastfeeding and gut microbiome is mostly evaluated using fecal samples. More studies are needed to investigate the link between SIBO and breastfeeding using breath tests.

Evidence shows that intestinal microflora is a crucial factor in immune system maturation and tolerance. ${ }^{34,35}$ Interestingly, a history of allergy is inversely correlated with the GBT profiles in Figure 2, which is contrary to previous studies. A recent study has shown that SIBO was linked to allergic disease. ${ }^{36}$ However, the study design was retrospective and involved a small number of subjects. Very few studies correlating SIBO and allergy have been reported. In 1 study, the prevalence of SIBO in patients with chronic urticaria was higher than in the healthy population, but the eradication of SIBO was not related to clinical improvements in patients with chronic urticaria. ${ }^{37} \mathrm{~A}$ well-designed prospective study with a larger number of patients is needed. The hygiene hypothesis in allergy suggests that exposure to enriched microbial environment improves the immune response in patients with allergic diseases. ${ }^{38}$ Although the breath test failed to detect the diversity of gut microbiota, the presence of bacteria demonstrated by breath test may indirectly reflect the beneficial status of allergic diseases. It is also possible that the SIBO negative results were caused by frequent overuse of antibiotics in patients with allergic diseases that manifested as upper respiratory tract infections. The antibiotic prescription rate is very high in Korea, where the study was conducted. According to data presented by the Organisation for Economic Co-operation and Development (OECD) in 2014, the rate of antibiotic consumption in Korea was 31.7 defined daily doses per 1000 inhabitants/day (DID), which is higher than the average rate of 20.5 DID in OECD countries. ${ }^{39}$ A future study is needed to validate the diagnosis of bacterial overgrowth based on breath tests and diversity of microbiota.

Loose stool can occur significantly in FAPD children with SIBO. We evaluated each gastrointestinal symptom using a validated questionnaire. Although the symptom scores of abdominal pain, hard stool, strain, urgency, bloating, flatus, satiety, frequent urination, and nausea were higher in GBT + compared with those of GBT - children, no significant differences were observed between the 2 groups. The foregoing results are based on a small sample size. The symptoms of hard stool and strain related to slow intestinal transit may be associated with $\mathrm{CH}_{4} \cdot{ }^{5,40}$ However, no pure $\mathrm{CH}_{4}$ excretors were detected in our study, and the clinical characteristics of subjects with $\mathrm{CH}_{4}$ gas related symptoms could not be investigated. The prevalence of $\mathrm{CH}_{4}$ excretors with age is known to be high, ranging from $3 \%$ in the 6-10 years old group to $45 \%$ in the 46-50 years old group. ${ }^{41}$ Thus, children with SIBO excreting $\mathrm{CH}_{4}$ constituted a relatively low proportion in our study.

In conclusion, SIBO in children and adolescents with FAPD 
was not uncommon. SIBO is a suspected therapeutic target for alleviating bowel symptoms of children older than 12 years manifesting functional gastrointestinal symptom such as loose stool. Further studies are needed to elucidate the role of SIBO in children and adolescents diagnosed with FAPD by demonstrating the potential response to antibiotic treatment.

Acknowledgements: This study was supported by the Korean Society of Pediatric Infectious Diseases. The authors are solely responsible for the content, which does not necessarily represent the official views of the Korean Society of Pediatric Infectious Diseases.

\section{Financial support: None.}

\section{Conflicts of interest: None.}

Author contributions: Jin Lee and Chang-Nyol Paik contributed to the study design, data interpretation, and writing of the manuscript; Jin Lee, Yeon-Ji Kim, Dae Bum Kim, Ji Min Lee, Sik Kyung Choi, Yeon Jong Cho, and Chang-Nyol Paik collected and interpreted the data; and Jin Lee, Chang-Nyol Paik, and Dae Bum Kim contributed to the study design and critically reviewed the manuscript. All authors approved the final draft.

\section{References}

1. Chitkara DK, Rawat DJ, Talley NJ. The epidemiology of childhood recurrent abdominal pain in Western countries: a systematic review. Am J Gastroenterol 2005;100:1868-1875.

2. Koppen IJ, Nurko S, Saps M, Di Lorenzo C, Benninga MA. The pediatric Rome IV criteria: what's new? Expert Rev Gastroenterol Hepatol 2017;11:193-201.

3. Korterink JJ, Benninga MA, van Wering HM Deckers-Kocken JM. Glucose hydrogen breath test for small intestinal bacterial overgrowth in children with abdominal pain-related functional gastrointestinal disorders. J Pediatr Gastroenterol Nutr 2015;60:498-502.

4. Avelar Rodriguez D, Ryan PM, Toro Monjaraz EM, Ramirez Mayans JA, Quigley EM. Small intestinal bacterial overgrowth in children: a state-of-the-art review. Front Pediatr 2019;7:363.

5. Lin HC. Small intestinal bacterial overgrowth: a framework for understanding irritable bowel syndrome. JAMA 2004;292:852-858.

6. Sieczkowska A, Landowski P, Zagozdzon P, Kaminska B, Lifschitz C. Small bowel bacterial overgrowth associated with persistence of abdominal symptoms in children treated with a proton pump inhibitor. J Pediatr 2015;166:1310-1312, e1.

7. Donowitz JR, Haque R, Kirkpatrick BD, et al. Small intestine bacterial overgrowth and environmental enteropathy in Bangladeshi children. mBio 2016;7:e02102-e02115.

8. Park JM, Choi MG, Oh JH, et al. Cross-cultural validation of irritable bowel syndrome quality of life in Korea. Dig Dis Sci 2006;51:1478-1484.
9. Park JM, Choi MG, Kim YS, et al. Quality of life of patients with irritable bowel syndrome in Korea. Qual Life Res 2009;18:435-446.

10. Lee JM, Lee KM, Chung YY, et al. Clinical significance of the glucose breath test in patients with inflammatory bowel disease. J Gastroenterol Hepatol 2015;30:990-994.

11. Longstreth GF, Thompson WG, Chey WD, Houghton LA, Mearin F, Spiller RC. Functional bowel disorders. Gastroenterology 2006;130:1480-1491.

12. Collins BS, Lin HC. Double-blind, placebo-controlled antibiotic treatment study of small intestinal bacterial overgrowth in children with chronic abdominal pain. J Pediatr Gastroenterol Nutr 2011;52:382-386.

13. de Boissieu D, Chaussain M, Badoual J, Raymond J, Dupont C. Smallbowel bacterial overgrowth in children with chronic diarrhea, abdominal pain, or both. J Pediatr 1996;128:203-207.

14. Siniewicz-Luzeúczyk K, Bik-Gawin A, Zeman K, Bąk-Romaniszyn L. Small intestinal bacterial overgrowth syndrome in children. Prz Gastroenterol 2015;1:28-32.

15. Ford AC, Spiegel BM, Talley NJ, Moayyedi P. Small intestinal bacterial overgrowth in irritable bowel syndrome: systematic review and metaanalysis. Clin Gastroenterol Hepatol 2009;7:1279-1286.

16. Sachdeva S, Rawat AK, Reddy RS, Puri AS. Small intestinal bacterial overgrowth (SIBO) in irritable bowel syndrome: frequency and predictors. J Gastroentero Hepatol 2011;26(suppl 3):135-138.

17. Pimentel M, Lin HC, Enayati P, et al. Methane, a gas produced by enteric bacteria, slows intestinal transit and augments small intestinal contractile activity. Am J Physiol Gastrointest Liver Physiol 2006;290:G1089-G1095.

18. Collins BS, Lin HC. Chronic abdominal pain in children is associated with high prevalence of abnormal microbial fermentation. Dig Dis Sci 2010;55:124-130.

19. Ghoshal UC, Shukla R, Ghoshal U. Small intestinal bacterial overgrowth and irritable bowel syndrome: a bridge between functional organic dichotomy. Gut Liver 2017;11:196-208.

20. Shah ED, Basseri RJ, Chong K, Pimentel M. Abnormal breath testing in IBS: a meta analysis. Dig Dis Sci 2010;5 5:2441-2449.

21. Chen B, Kim JJ, Zhang Y, Du L, Dai N. Prevalence and predictors of small intestinal bacterial overgrowth in irritable bowel syndrome: a systematic review and meta-analysis. J Gastroenterol 2018;53:807-818.

22. Gasbarrini A, Corazza GR, Gasbarrini G, et al. Methodology and indications of $\mathrm{H}_{2}$-breath testing in GI diseases: the Rome Consensus Conference.Aliment Pharmacol Ther 2009;29(suppl 1):1-49.

23. Rezaie A, Buresi M, Lembo A, et al. Hydrogen and methane-based breath testing in gastrointestinal disorders: the north American consensus. Am J Gastroenterol 2017;112:775-784.

24. Simrén M, Stotzer PO. Use and abuse of hydrogen breath tests. Gut 2006;55:297-303.

25. Sellin JH, Hart R. Glucose malabsorption associated with rapid intestinal transit. Am J Gastroenterol 1992;87:584-589.

26. Walters B, Vanner SJ. Detection of bacterial overgrowth in IBS using the lactulose $\mathrm{H} 2$ breath test: comparison with 14C-D-xylose and healthy controls. Am J Gastroenterol 2005;100:1566-1570.

27. Riordan SM, McIver CJ, Walker BM, Duncombe VM, Bolin TD, 
Thomas MC. The lactulose breath hydrogen test and small intestinal bacterial overgrowth. Am J Gastroenterol 1996;91:1795-1803.

28. Bond JH Jr, Levitt MD, Prentiss R. Investigation of small bowel transit time in man utilizing pulmonary hydrogen $\left(\mathrm{H}_{2}\right)$ measurements. J Lab Clin Med 1975;85:546-555.

29. Nam KC, Jo C, Lee M. Meat products and consumption culture in the East. Meat Sci 2010;86:95-102.

30. Gwee KA, Bak YT, Ghoshal UC, et al. Asian consensus on irritable bowel syndrome. J Gastroenterol Hepatol 2010;25:1189-1205.

31. Yu D, Cheeseman F, Vanner S. Combined oro-caecal scintigraphy and lactulose hydrogen breath testing demonstrate that breath testing detects oro-caecal transit, not small intestinal bacterial overgrowth in patients with IBS. Gut 2011;60:334-340.

32. Choung RS, Ruff KC, Malhotra A, et al. Clinical predictors of small intestinal bacterial overgrowth by duodenal aspirate culture. Aliment Pharmacol Ther 2011;33:1059-1067.

33. Akagawa S, Tsuji S, Onuma C, et al. Effect of delivery mode and nutrition on gut microbiota in neonates. Ann Nutr Metab 2019;74:132-139.

34. Gourbeyre P, Denery S, Bodinier M. Probiotics, prebiotics, and synbiotics: impact on the gut immune system and allergic reactions. J Leukoc Biol 2011;89:685-695.

35. Nakayama J, Kobayashi T, Tanaka S, et al. Aberrant structures of fecal bacterial community in allergic infants profiled by $16 \mathrm{~S}$ rRNA gene pyrosequencing. FEMS Immunol Med Microbiol 2011;63:397-406.

36. Peña-Vélez R, Toro-Monjaraz E, Avelar-Rodríguez D, et al. Small intestinal bacterial overgrowth: could it be associated with chronic abdominal pain in children with allergic diseases? Rev Esp Enferm Dig 2019;111:927-930.

37. Campanati A, Gesuita R, Giannoni M, et al. Role of small intestinal bacterial overgrowth and Helicobacter pylori infection in chronic spontaneous urticaria: a prospective analysis. Acta Derm Venereol 2013;93:161164.

38. Untersmayr E, Bax HJ, Bergmann C, et al. AllergoOncology: microbiota in allergy and cancer-a European academy for allergy and clinical immunology position paper. Allergy 2019;74:1037-1051.

39. Organisation for Economic Co-operation and Development. Antibiotic consumption levels, Paris. Available from URL: https:/www.oecd.org/ health/antimicrobial-resistance.htm (accessed 22 Nov 2021).

40. Pimentel M, Chow EJ, Lin HC. Normalization of lactulose breath testing correlates with symptom improvement in irritable bowel syndrome. a double-blind, randomized, placebo-controlled study. Am J Gastroenterol 2003;98:412-419.

41. Polag D, Leiß O, Keppler F. Age dependent breath methane in the German population. Sci Total Environ 2014;481:582-587. 